\title{
Spatial variation of pneumonia hospitalization risk in Twin Cities metro area, Minnesota
}

\author{
P. Y. IROH TAM ${ }^{1,2 * \dagger}$, B. KRZYZANOWSKI ${ }^{3} \dagger$, J. M. OAKES ${ }^{4}, \mathrm{~L} \mathrm{KNE}^{3,5}$ AND \\ S. MANSON ${ }^{3}$ \\ ${ }^{1}$ Division of Pediatric Infectious Diseases and Immunology, Department of Pediatrics, University of Minnesota \\ Medical School, Minneapolis, MN, USA \\ ${ }^{2}$ Malawi-Liverpool Wellcome Trust Clinical Research Programme, Blantyre, Malawi \\ ${ }^{3}$ Department of Geography, Environment, and Society, University of Minnesota, Minneapolis, MN, USA \\ ${ }_{5}^{4}$ Department of Epidemiology, University of Minnesota School of Public Health, Minneapolis, MN, USA \\ ${ }^{5}$ U-Spatial, Research Computing, Office of Vice President for Research, University of Minnesota, Minneapolis, \\ $M N, U S A$
}

Received 3 February 2017; Final revision 11 September 2017; Accepted 12 September 2017; first published online 17 October 2017

\section{SUMMARY}

Fine resolution spatial variability in pneumonia hospitalization may identify correlates with socioeconomic, demographic and environmental factors. We performed a retrospective study within the Fairview Health System network of Minnesota. Patients 2 months of age and older hospitalized with pneumonia between 2011 and 2015 were geocoded to their census block group, and pneumonia hospitalization risk was analyzed in relation to socioeconomic, demographic and environmental factors. Spatial analyses were performed using Esri's ArcGIS software, and multivariate Poisson regression was used. Hospital encounters of 17840 patients were included in the analysis. Multivariate Poisson regression identified several significant associations, including a $40 \%$ increased risk of pneumonia hospitalization among census block groups with large, compared with small, populations of $\geqslant 65$ years, a $56 \%$ increased risk among census block groups in the bottom (first) quartile of median household income compared to the top (fourth) quartile, a $44 \%$ higher risk in the fourth quartile of average nitrogen dioxide emissions compared with the first quartile, and a $47 \%$ higher risk in the fourth quartile of average annual solar insolation compared to the first quartile. After adjusting for income, moving from the first to the second quartile of the race/ethnic diversity index resulted in a $21 \%$ significantly increased risk of pneumonia hospitalization. In conclusion, the risk of pneumonia hospitalization at the censusblock level is associated with age, income, race/ethnic diversity index, air quality, and solar insolation, and varies by region-specific factors. Identifying correlates using fine spatial analysis provides opportunities for targeted prevention and control.

Key words: Spatial modeling, pneumonia, infectious diseases epidemiology, lower respiratory tract infections.

\footnotetext{
* Author for correspondence: P. Y. Iroh Tam, Malawi-Liverpool Wellcome Trust Clinical Research Programme, P.O. Box 30096, Chichiri, Blantyre 3, Malawi.

(Email: irohtam@mlw.mw)

$\dagger$ Both authors contributed equally to this work.
}

\section{INTRODUCTION}

Pneumonia is a cause of enormous morbidity and mortality worldwide, including in the USA where it accounts for $4 \cdot 2$ million clinic visits and over 60000 
deaths in adults, and an economic burden of over $\$ 17$ billion, annually [1]. Pneumonia is a leading cause of pediatric hospitalization and is the eighth leading cause of death in this country $[2,3]$. While pneumococcal conjugate vaccines have been effective in decreasing rates of pneumonia hospitalizations nationally [4, 5] as well as in Minnesota [6], sustained decreases were noted only in the younger children receiving the vaccine [6], suggesting that exposures beyond immunization are affecting disease and hospital admission.

Pneumonia and pneumonia hospitalization have a long-recognized correlation with socioeconomic risk factors. Adult bacteremic pneumococcal pneumonia and infant risk of developing pneumonia have both been associated with lower income and education [7]. Similarly, pneumonia hospitalization rates have been significantly associated with socioeconomic deprivation $[8,9]$, and geographic variation in pneumonia hospitalization has been associated with underlying socioeconomic conditions [8]. However, whether spatial variability is also linked to other demographic and environmental factors beyond socioeconomic status and education is not well described.

Spatial modeling of infection can define distinct, local patterns for disease incidence and local covariation with social and environmental factors, and thereby identify targets for implementation of public health strategies. A fine spatial resolution such as to the block-group level can allow for a refined level of analysis of pneumonia hospitalizations that can provide a more accurate depiction of the underlying data, and has been explored using postcode districts [10], census divisions [9], and aggregated census tracts [7]. Spatial variation is a tool that could be used to identify some of these environmental factors in specific areas or population groups that might not be easily detected with other designs. We hypothesized that use of this approach would identify whether spatial variability exists and could correlate pneumonia hospitalizations with socioeconomic, demographic and environmental factors. The objective of this study was to evaluate if location-specific patterns of pneumonia, and socioeconomic, demographic and environmental factors can exist within a relatively small geographic region.

\section{METHODS}

This was a cross-sectional study design of patients within the Fairview Health System network of
Minnesota. The Fairview Health System network comprises eight hospitals in the seven-county metro area of the Twin Cities encompassing 7706.5 square kilometers, including six community hospitals, a 96-bed tertiary care children's hospital and an 874-bed tertiary care university hospital. The clinical data repository includes data from 12.2 million outpatient visits, 514000 inpatient visits, and 333000 Emergency Department visits in 2015. During the study period, roughly $20 \%$ of the metro area's population was hospitalized within the network.

All patients 2 months of age and older hospitalized within the network between 1 January 2011 and 8 November 2015 with a diagnosis of pneumonia were identified. We identified participants through International Classification of Diseases, Ninth Edition (ICD-9) and Tenth Edition (ICD-10) codes diagnosed with pneumonia, pneumococcal or streptococcal septicemia. Patients with home addresses beyond the seven-county metro area (Anoka, Carver, Dakota, Hennepin, Ramsey, Scott, and Washington Counties), seen in the outpatient clinic, or who were re-hospitalized within a month were excluded. Variables extracted included age, sex, race/ ethnicity, diagnoses and residential address. This study was approved by the Institutional Review Board of the University of Minnesota.

Patients were geocoded to their census block group by linkage to their residential address. Vague and incomplete addresses (e.g. P.O. boxes, General Delivery) were excluded. Primary exposures were obtained from Esri's Business Analyst [11], American Fact Finder [12], the Minnesota Solar Suitability Analysis [13], and the Marshall Research Group [14], using 5 year estimates from 2010 to 2014. Covariates for analysis included median household income, unemployment rate, educational attainment, living below the poverty line, racial and ethnic diversity index [15], average annual air quality (amount of nitrogen dioxide $\left(\mathrm{NO}_{2}\right)$ measured in parts per billion), and average annual solar insolation (measured in kilowatt-hour per meter squared $(\mathrm{kWh} /$ $\left.\mathrm{m}^{2}\right)$ ). The primary outcome of interest was census block group-level pneumonia hospitalization risk, which was calculated for each block group using the numerator as the number of cases present within a block group, and the denominator as the total population within the block group. For the age analysis, rather than assume that the patient population is equally distributed, we created a new variable at block group level based on census data: high and 
low number of individuals aged $\geqslant 65$ (the top 50\%, compared with the bottom $50 \%$, of the distribution of number of individuals aged $\geqslant 65$ years). Seasons were defined according to the National Oceanic and Atmospheric Administration as spring (MarchMay), summer (June-August), fall (SeptemberNovember) and winter (December-February) [16].

In order to promote ease of interpretation and preserve nonlinear trends, all variables were defined by quartiles. Educational attainment was defined by the number of college-educated individuals per block group (those who have attained a Bachelor's degree or higher). Average annual solar insolation was transformed to represent kilowatt-hour per square meter $\left(\mathrm{kWh} / \mathrm{m}^{2}\right)$, dividing average annual solar insolation per block group by the area of the block group.

Statistical analyses were performed using STATA software, v.13 (StataCorp, College Station, TX). Spatial analyses were performed using Esri's ArcGIS software, v.10.4 (Esri, Redlands, CA). To correct for positive or negative spatial variability between the observed values and their distances, we utilized Moran's I statistic in assessing the degree of spatial autocorrelation [17]. A multivariate Poisson regression was used to test for differences and associations between pneumonia hospitalization rates over block group with socioeconomic, demographic and environmental variables. Cluster spatial analysis was performed using the Getis-Ord Gi* statistic [18], which characterized hot or cold block group clusters with higher or lower than expected pneumonia hospitalization rates, respectively. These hot and cold spots were overlaid with the distribution of socioeconomic, demographic, and environmental variables to assess for significant associations. Geographic masking was performed on all maps in order to promote comparability.

\section{RESULTS}

A total of 18362 patients were identified, and 17840 patients ranging from 10 weeks to 105 years (median age 66 years) were included in the analysis. Fifty-one percent were female. The majority of the patients were white $(82 \%), 8.7 \%$ black, $1.8 \%$ Hispanic, and $6.8 \%$ other. Average block group population was 1416 (range 267- 8965) with an average block group area of $7366872 \mathrm{~km}^{2}$ (range 81 251-303 581842 $\mathrm{km}^{2}$ ). The bottom $50 \%$ of block groups with a population $\geqslant 65$ years ranged from 0 to 148 persons, whereas the top $50 \%$ of block groups ranged from
149 to 1232 persons. A small proportion (196 block groups, or $9.4 \%$ ) within the Twin Cities seven-county metro area had no pneumonia hospitalizations documented in the Fairview Health System. Among the other 1889 block groups with cases, pneumonia hospitalization risk ranged from $0.02 \%$ to $8.05 \%$ (Fig. 1a). The calculated average pneumonia hospitalization risk within a block group was 6 cases per every 1000 persons.

According to the Poisson analysis, those residing within block groups containing the bottom (first) quartile of the distribution of median household income had a 56\% (Incidence rate ratio (IRR) 1.56 , 95\% confidence interval (CI) (1.31-1.85)) increased risk of pneumonia hospitalization compared with those at the top (fourth) quartile of the income distribution (Fig. 1b; online supplementary Appendix Table). No significant difference was detected between the first and second quartiles of the distribution of median household income (IRR 0.98, 95\% CI (0.811·18)). Arguably, the decreased risk observed between the first and third quartile of income was marginally significant (IRR $0 \cdot 85,95 \%$ CI $(0 \cdot 70-1 \cdot 02)$ ), and a trend was apparent in the data where increases in income accompanied a decrease in risk. Among the distribution of a number of individuals living below the poverty line, pneumonia hospitalization risk increased approximately 19\% (IRR $1 \cdot 19,95 \%$ CI (1.01-1.41)) going from the first to the fourth quartile. Furthermore, the risk of pneumonia hospitalization increased with higher rates of unemployment rates among block groups: from the first quartile of the distribution of unemployment rate to the third and fourth quartiles, pneumonia hospitalization risk increased approximately $20 \%$ for both (IRR 1.20, 95\% CI (1.03-1.41); and $1 \cdot 22$, 95\% CI $(1 \cdot 03-1 \cdot 46)$, respectively).

After adjusting for median household income, there was a $39 \%$ increased the risk of pneumonia hospitalization among those in the second quartile of the distribution of college-educated individuals relative to those in the first quartile (IRR $1 \cdot 39,95 \%$ CI $(1 \cdot 18$ 1.64)). When going from the first to the third and fourth quartiles among college-educated individuals, the risk of pneumonia hospitalization increased $64 \%$ (IRR $1 \cdot 64,95 \%$ CI $(1 \cdot 38-1 \cdot 96)$ ) and 61\% (IRR 1.61, 95\% CI (1.28-2.02)), respectively. Similarly, after adjusting for median household income among the uninsured, there was a $21 \%$ (IRR $0.79,95 \%$ CI $(0.67-0.94))$ decreased risk of pneumonia hospitalization for those in the second, and a 24\% (IRR 0.76, 
(a)

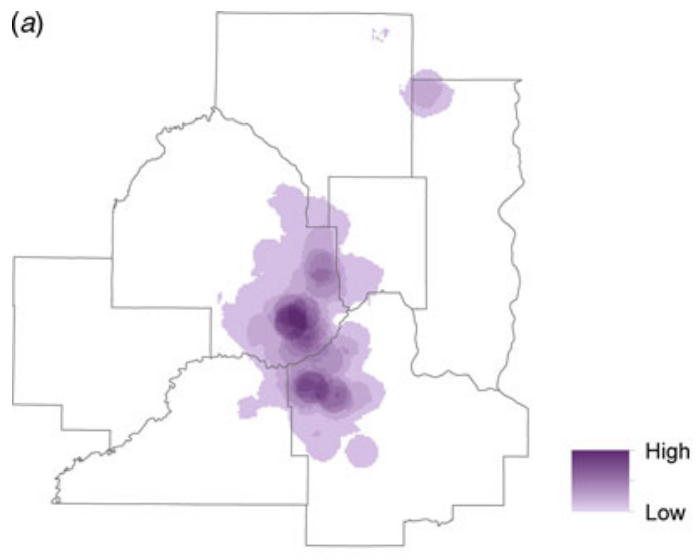

(c)

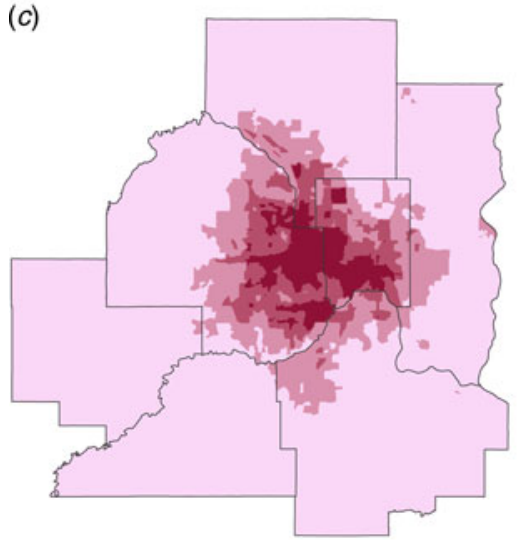

(b)

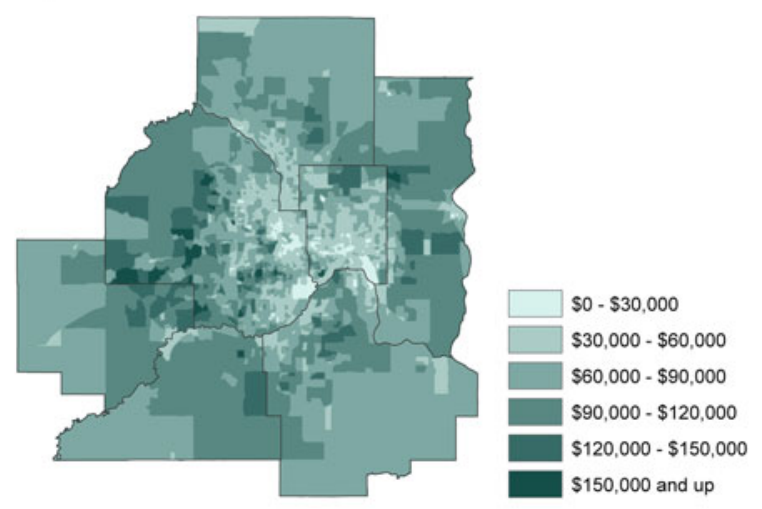

(d)

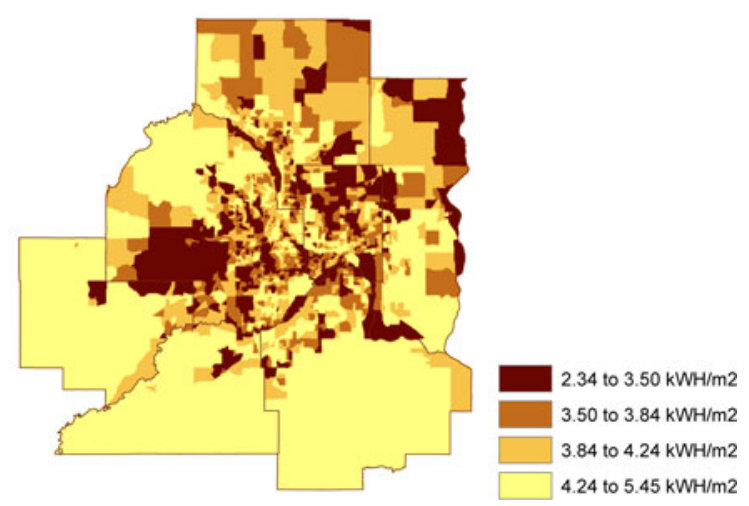

Fig. 1. 2011-2015 Twin Cities seven-county metro area by block group of: (a) Pneumonia hospitalization risk (block group cases per block group population); (b) Median household income (dollars); (c) Average $\mathrm{NO}_{2}$ emission quartiles (ppb, parts per billion); (d) Solar insolation quartiles $\left(\mathrm{kWh} / \mathrm{m}^{2}\right)$. Geographic masking was performed on all maps in order to promote comparability.

95\% CI $(0 \cdot 62-0 \cdot 92))$ decreased risk for those in the fourth, compared with the first, quartiles.

A significant association was found across all quartiles of race/ethnic diversity index. When moving from the first to the second and third quartiles, the risk of pneumonia hospitalization increased by $31 \%$ (IRR $1 \cdot 31,95 \%$ CI $(1 \cdot 12-1 \cdot 53))$ and $34 \%$ (IRR $1 \cdot 34,95 \%$ CI (1.13-1.58)), respectively. Moving from the first (lowest) to the fourth (highest) quartile of the race/ethnic diversity index led to a $44 \%$ (IRR $1 \cdot 44,95 \%$ CI $(1 \cdot 22-1 \cdot 71))$ increase in pneumonia hospitalization risk. After adjusting for income, a significant association was found only between the first and second quartiles of race/ethnic diversity index, where moving from the first to the second quartile resulted in a $21 \%$ (IRR $1 \cdot 21,95 \%$ CI (1.04-1.41)) increased the risk of pneumonia hospitalization.

There were significant differences in pneumonia hospitalization by age group. Block groups with larger populations of adults $\geqslant 65$ years had pneumonia hospitalization rates that were $40 \%$ (IRR $1 \cdot 40,95 \%$ CI $(1 \cdot 26-1 \cdot 56))$ higher than the block groups containing smaller populations of older adults.

Seasonal analysis also revealed significant differences in pneumonia hospitalization by age group. For all seasons, block groups containing a larger population of adults $\geqslant 65$ years had greater pneumonia hospitalization rates than the block groups containing smaller populations of older adults (Table 1).

In examining environmental factors, air quality was found to have a significant relationship with pneumonia hospitalizations across all quartiles of the distribution of average annual $\mathrm{NO}_{2}$ emissions. When going from the first to the second and third quartiles, the risk of pneumonia hospitalization increased $56 \%$ (IRR $1 \cdot 56,95 \%$ CI $(1 \cdot 32-1 \cdot 84)$ ) and 37\% (IRR $1 \cdot 37$, 95\% CI $(1 \cdot 16-1 \cdot 60))$, respectively (Fig. 1c). Block groups containing the highest quartile of average 
Table 1. Incidence rate ratio and risk of pneumonia hospitalization by season for block groups containing a larger population of adults $\geqslant 65$ years

\begin{tabular}{llll}
\hline \hline & IRR $(95 \% \mathrm{CI})$ & Risk per 1000 persons per year & $P$ value \\
\hline Spring (March-May) & $1 \cdot 39(1 \cdot 23-1 \cdot 58)$ & $1 \cdot 24$ & $<0 \cdot 001$ \\
Summer (June-August) & $1.39(1 \cdot 22-1 \cdot 58)$ & 1.04 & $<0 \cdot 001$ \\
Autumn (September-November) & $1.31(1 \cdot 15-1 \cdot 49)$ & 1.35 & $<0 \cdot 001$ \\
Winter (December-February) & $1 \cdot 43(1 \cdot 27-1 \cdot 63)$ & $1 \cdot 26$ & $<0 \cdot 001$ \\
\hline \hline
\end{tabular}

CI, confidence interval; IRR, incidence rate ratio.

Block groups containing smaller populations of $\geqslant 65$ years are the reference group.

$\mathrm{NO}_{2}$ emissions had a hospitalization rate for pneumonia that was $44 \%$ (IRR $1 \cdot 44,95 \%$ CI $(1 \cdot 22-1 \cdot 69)$ ) higher than the lowest quartile.

Several statistically significant interactions were detected between age group and air quality (Fig. 2). Block groups containing larger populations of adults $\geqslant 65$ years who live in regions with the second, third, and fourth quartiles of $\mathrm{NO}_{2}$ emissions had $68 \%$ (IRR 1.68, 95\% CI (1.37-2.06)), 58\% (IRR $1 \cdot 58,95 \%$ CI $(1 \cdot 28-1 \cdot 96))$ and $110 \%$ (IRR $2 \cdot 10,95 \%$ CI (1.64-2.71)) higher risk for pneumonia hospitalization, respectively, than those living in block groups with the lowest quartile of average annual $\mathrm{NO}_{2}$. Comparing the lowest quartile of $\mathrm{NO}_{2}$ emissions to the highest, for block groups containing smaller populations of older adults, was only marginally significant (IRR 1·22, 95\% CI (1·00-1·48)).

Statistically significant interactions between age group and air quality remained after stratifying by a season where, for all four seasons, block groups containing larger populations of adults $\geqslant 65$ years in regions with higher $\mathrm{NO}_{2}$ emissions had a greater risk for pneumonia hospitalization than those from block groups with the lowest average annual $\mathrm{NO}_{2}$ (Fig. 3).

Furthermore, strong and significant associations were detected between solar insolation and pneumonia hospitalization risk, where regions with higher relative to lower average annual solar insolation also had a greater risk of pneumonia hospitalization. Moving from the first to the second quartile of solar insolation resulted in a $16 \%$ (IRR $1 \cdot 16,95 \% \mathrm{CI}$ $(0 \cdot 99-1 \cdot 35))$ increased the risk of pneumonia hospitalization. Moving from the first to the third and fourth quartiles of average annual solar insolation the risk of pneumonia hospitalization increased by $19 \%$ (IRR $1 \cdot 19,95 \%$ CI (1.03-1·38)) and 47\% (IRR $1 \cdot 47,95 \%$ CI (1.25-1.73)), respectively (Fig. 1d).

There was significant geographic clustering as suggested by the results of the Getis Ord Hot-Spot

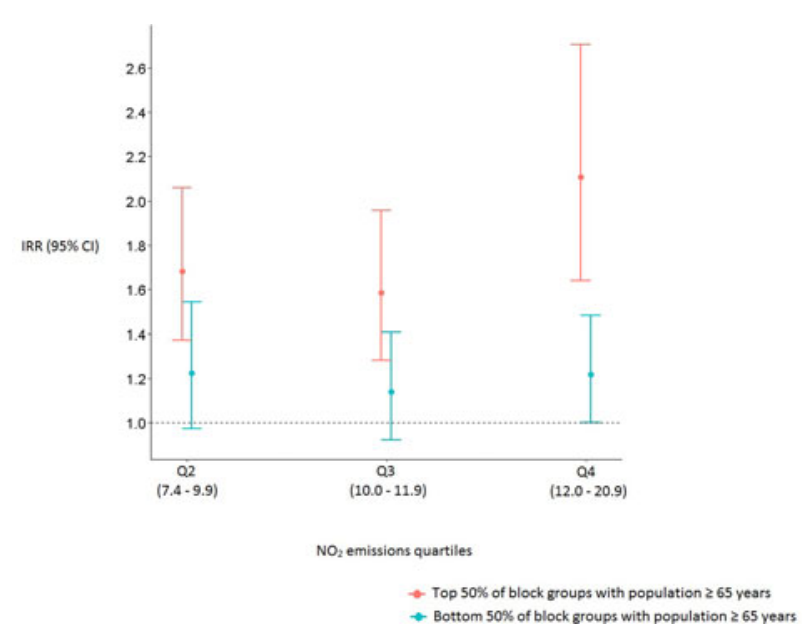

Fig. 2. Incidence rate ratios and $95 \%$ confidence intervals of pneumonia hospitalizations for block groups with larger and smaller populations of $\geqslant 65$ years. The first quartile of average annual $\mathrm{NO}_{2}$ emissions serves as the reference group.

Analysis (Fig. 4). The hot-spot analysis showed spatial correspondence to the expected relationships: hot spots (higher than expected pneumonia hospitalization rates) were situated in areas where higher levels of $\mathrm{NO}_{2}$ emissions or lower average income were apparent, while cold spots (lower than expected pneumonia hospitalization rates) were situated in the higher income areas characterized by lower levels of $\mathrm{NO}_{2}$ emissions.

\section{DISCUSSION}

Using data at the fine spatial resolution of the block group, this study identified significant spatial variability in pneumonia hospitalization risk across the Twin Cities seven-county metro area in Minnesota. This variability was associated with underlying distributions of socioeconomic, demographic and environmental factors such as measures of income, race/ 

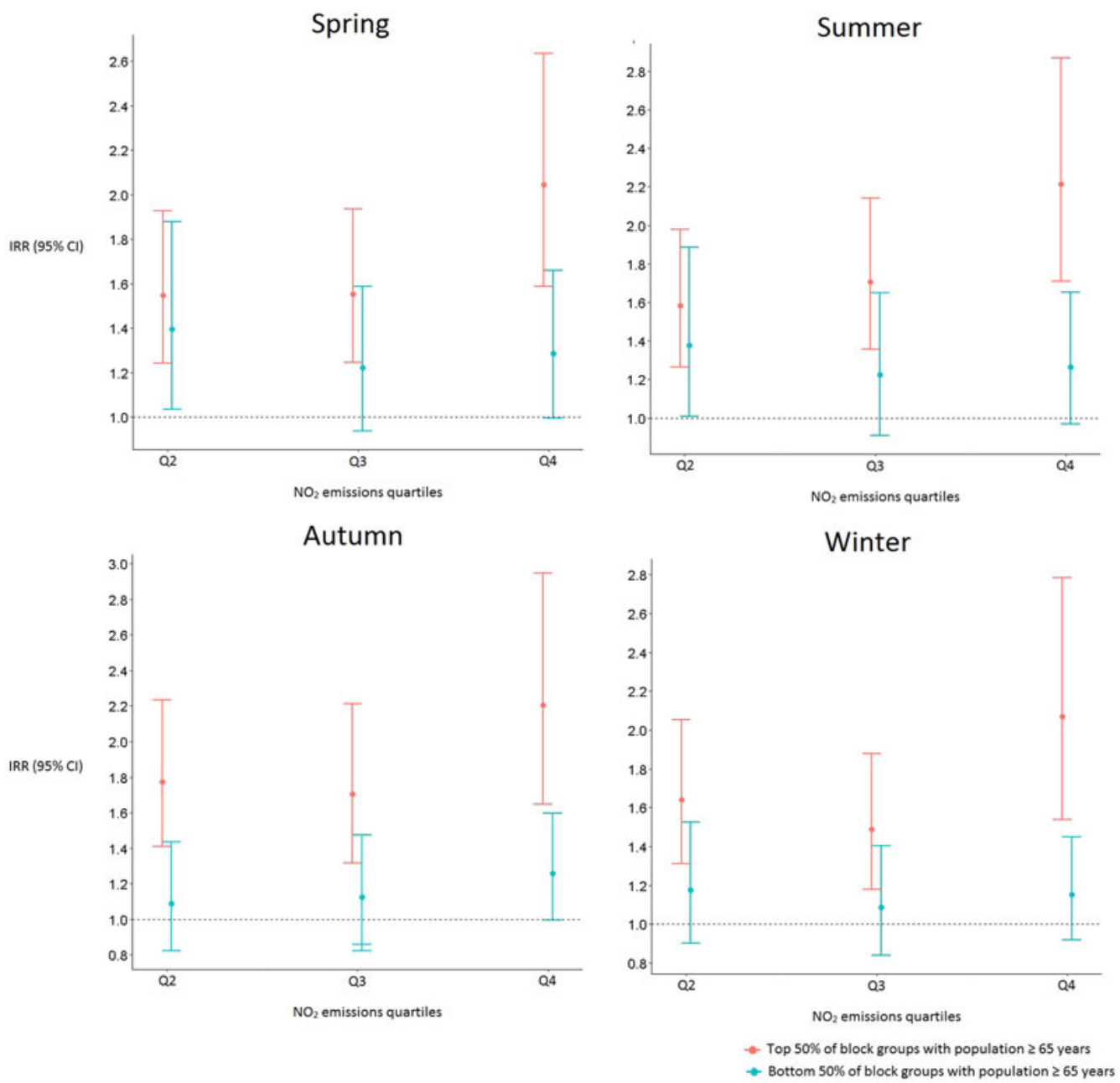

Fig. 3. Incidence rate ratios and $95 \%$ confidence intervals of pneumonia hospitalization by season for block groups with larger and smaller populations of $\geqslant 65$ years. The first quartile of average annual $\mathrm{NO}_{2}$ emissions serves as the reference group.

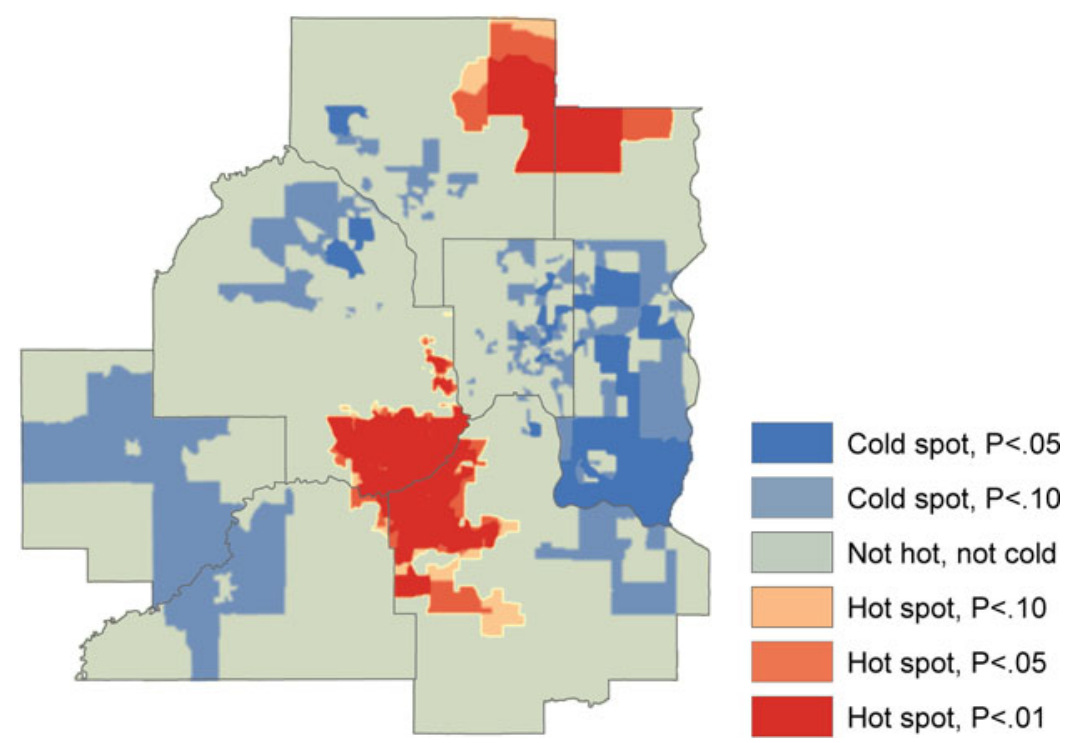

Fig. 4. Hot-spot analysis for significant spatial clusters of pneumonia hospitalization, 2011-2015. 
ethnic diversity index, unemployment, poverty, air quality, and solar insolation. While these findings are broadly consistent with previous studies on the association between increased risk of respiratory infections and lower socioeconomic status, and we also report significant associations with lesser described factors.

Previous studies have reported on spatial variation in health, including the poorer health indices [19-22] and increased respiratory illness $[8,10,23-26]$ that are seen in lower-income neighborhoods and communities. Spatial associations with respiratory tract infections in areas with lower socioeconomic status have been attributed to higher population density [20], increased environmental particle exposure and pollutants in these areas [27], and limited access to health care [28]. Findings of documented variability have been posited to inform resource-delivery to promote hospitalization prevention [29].

In our study, contrary to expected, greater risk of pneumonia hospitalization was significantly associated with increased numbers of college-educated individuals per block group. One explanation is that college-educated individuals are more likely to seek clinical care than those without college degrees. The inclusion of median household income in our model gave rise to unstable effect estimates as a result of the undeniable collinearity that persists between race/ethnic diversity index and income. Because it is nearly impossible to piece apart from the independent effects of race/ethnic diversity index and income, one should interpret the adjusted model with caution. Counter to what one would expect, our data suggests that as the number of uninsured individuals per block group increases, the risk of pneumonia hospitalization decreases. Because we relied on hospital admissions for our risk calculation, we are missing data from those who had pneumonia and did not seek clinical care. Therefore, one explanation for our observations is that individuals who are uninsured are less likely to go to the hospital when sick as they are deterred from the cost of care. Block groups with larger populations of $\geqslant 65$ years had significantly higher pneumonia hospitalization rates compared with block groups with smaller populations of $\geqslant 65$ years, which correlates with what is known about higher morbidity occurring in this population. The significant interaction between air quality and age $\geqslant 65$ years suggests that this group may be vulnerable to pneumonia hospitalization induced by poor air quality. Block groups containing larger populations of $\geqslant 65$ years in regions with higher
$\mathrm{NO}_{2}$ emissions had a greater risk for pneumonia hospitalization than those from block groups with the lower average annual $\mathrm{NO}_{2}$, across all seasons, suggesting that vulnerability of this group to the pneumoniainducing effects of air quality exists regardless of the season.

Air quality has been identified as a risk factor for childhood pneumonia and mortality in low- and middle-income countries [30]. In the United States, levels of ambient air pollutants have been associated with death rates from pneumonia [31] and air pollution has been linked to childhood respiratory health in rural Alaska Native communities [32]. However, much of the literature has focused on childhood illness and also has not specifically examined the impact of air quality on hospitalization rates. Of two studies that did, one demonstrated that coarse particulate matter was associated with increased risk of emergency hospital pneumonia admissions in Hong Kong [33]; another study revealed an association between increased particulate exposure and pneumonia admissions among the elderly [34].

Similarly, solar insolation has been explored in studies evaluating the association between ultraviolet $\mathrm{B}$ radiation and vitamin $\mathrm{D}$ on a variety of dermatologic, neurodevelopmental, endocrine, oncologic and autoimmune conditions [35]. While the potential association with pneumonia was described in ecological studies [36], including one performed in the United States where solar ultraviolet B radiation doses reduced the influenza fatality rate during 1918-1919 pandemic influenza [37], this is to our knowledge the first study to show an association with pneumonia hospitalizations. Our association of high solar insolation with increased pneumonia hospitalization was surprising and contrary to previous findings. In these studies, solar insolation was linked to a decrease in respiratory tract illness, which was attributed to the biosynthesis of vitamin D and its associated antioxidant properties and antibacterial activity $[38,39]$. In the presence of sunlight or UV rays, certain pollutants may undergo chemical reactions. Therefore, solar radiation may increase toxic by-products or may release pollutants such as trapped nitrogen oxide. As we do not have a complete understanding of the factors involved in the significant relationship between solar insolation and pneumonia hospitalization, it is difficult to say for certain what the data is showing. We controlled for income and education in our model. We can speculate that the relationship detected between solar insolation and 
pneumonia hospitalization reflects the association of $\mathrm{NO}_{2}$ emissions with risk by the way of road network density [40]. Possibly, block groups with greater solar insolation have greater road network density and subsequently higher annual $\mathrm{NO}_{2}$ emissions, and therefore greater pneumonia hospitalization risk. Another possible relationship is that ground-level ozone and other secondary pollutants can form when $\mathrm{NO}_{2}$ and volatile organic compounds mix due to sunlight [41]. Solar insolation is clearly a topic of future research given the various potential illness pathways it presents.

Spatial variation of pneumonia hospitalizations has been explored using postcode districts [10], census divisions [9], and aggregated census tracts [7]. However, these studies explored the spatial variation of pneumonia on larger areas of interest using coarser spatial resolutions. A study of pneumonia hospitalization at the county level identified geographic variation in risk, but this focused only on children [8]. By using spatial resolution to the block group level, our examination of pneumonia hospitalizations allowed for a refined level of analysis that can provide more accurate depiction of the underlying data [23], as well as capture relationships among population, socioeconomic and demographic factors, health and the environment that vary with fine-scaled differences in land use and housing.

Our sample size included almost 18000 patient encounters which are, to our knowledge, the largest study performed to date that has used fine resolution spatial analysis to examine pneumonia hospitalizations. However, the Fairview Health System network accounted for only about $20 \%$ of hospitalizations within the Twin Cities seven-county metro area during this study period, which limits the capture of a population-wide perspective on spatial patterns. Despite this, the system was distributed fairly widely, which is supported by the fact that $90.6 \%$ of block groups reported some level of pneumonia hospitalization. This would support the argument that the sample is spatially broad and geographically distributed and, from that, likely representative of the population. Another limitation is that pneumonia hospitalizations were analyzed across space but not over time, and thus our results do not account for seasonal effects on the risk of pneumonia hospitalization. We did not stratify our analysis by age group, which may have revealed additional associations. Given that our study was cross-sectional in design, we cannot make causal conclusions regarding any spatial patterns observed, and hence findings cannot be generalized to larger spatial scales.

In conclusion, we utilized a novel approach to evaluate relationships between pneumonia hospitalization and sociodemographic and environmental factors. We demonstrated that the risk of pneumonia hospitalization at the census block level varies by region-specific socioeconomic and environmental factors such as income, race/ethnic diversity index, unemployment, poverty, air quality and solar insolation. Using fine spatial resolution to the block group level we describe new associations which merit further study, and demonstrate proof-of-concept of an approach that may help identify high-risk regions that would most benefit from targeted interventions and prevention strategies [20,29]. Varying spatial units can have different pneumonia hospitalization correlations with socioeconomic, demographic, and environmental factors. Future studies should compare the various spatial units and their correlations.

\section{SUPPLEMENTARY MATERIAL}

The supplementary material for this article can be found at https://doi.org/10.1017/S0950268817002291

\section{ACKNOWLEDGEMENTS}

We thank Gretchen Sieger for identification of medical records for this study. We thank Richard MacLehose and Julian Wolfson for biostatistical support, Coleman Shepard for extracting the solar data, Josh Donato for writing the script to extract the solar data, Tracy Kugler for assistance preparing the figures, and David Van Riper for guidance on masking techniques. We thank Zohara Cohen, Lori Ketola and Amanda Bailor for assistance on addressing health information privacy. Research reported in this publication was supported by the University of Minnesota Institute for Health Informatics Seed Grant and the National Center for Advancing Translational Sciences of the National Institutes of Health Award Number UL1TR000114. This work was also supported in part by NSF OCI: Terra Populus: A Global Population/Environment Data Network (0940818), the National Institutes of Health supported Minnesota Population Center (R24 HD041023), and the University of Minnesota Office of the Vice President for Research. We gratefully acknowledge support from the Minnesota Population Center, which is funded by a center 
grant from the National Institutes of Health (P2C HD041023). The content is solely the responsibility of the authors and does not necessarily represent the official views of the National Institutes of Health.

\section{REFERENCES}

1. File TM Jr., Marrie TJ. Burden of community-acquired pneumonia in North American adults. Postgraduate Medicine 2010; 122(2): 130-141.

2. Keren R, et al. Prioritization of comparative effectiveness research topics in hospital pediatrics. Archives of Pediatrics \& Adolescent Medicine 2012; 166(12): 11551164.

3. National Center for Health Statistics. National Vital Statistics Report. Deaths: Final Data for 2006, vol. 57. 2009.

4. Griffin MR, et al. U.S. hospitalizations for pneumonia after a decade of pneumococcal vaccination. New England Journal of Medicine 2013; 369(2): 155-163.

5. Grijalva CG, et al. Decline in pneumonia admissions after routine childhood immunisation with pneumococcal conjugate vaccine in the USA: a time-series analysis. Lancet 2007; 369(9568): 1179-1186.

6. Iroh Tam PY, Koopmeiners JS. Trends in pneumonia hospitalizations in Hennepin County, Minnesota, 1999-2010. Mayo Clinic Proceedings 2013; 88(10): 1181-1182.

7. Thorn LK, et al. Pneumonia and poverty: a prospective population-based study among children in Brazil. $B M C$ Infectious Diseases 2011; 11: 180.

8. Beck AF, et al. Geographic variation in hospitalization for lower respiratory tract infections across one county. JAMA Pediatrics 2015; 169(9): 846-854.

9. Crighton EJ, et al. A spatial analysis of the determinants of pneumonia and influenza hospitalizations in Ontario (1992-2001). Social Science \& Medicine 2007; 64(8): 1636-1650.

10. Blain AP, et al. Spatial variation in the risk of hospitalization with childhood pneumonia and empyema in the North of England. Epidemiology and Infection 2014; 142 (2): $388-398$

11. Anon. Esri's Business Analyst (http://www.esri.com/ software/businessanalyst). Accessed 23 September 2016.

12. Anon. American Fact Finder (https://factfinder. census.gov/faces/nav/jsf/pages/index.xhtml). Accessed 23 September 2016.

13. Brink C, et al. Solar insolation, Minnesota (2006-2012) (http://dx.doi.org/10.13020/D6X59X). Accessed 6 January 2017.

14. Anon. Marshall Research Group (http://personal.ce. umn.edu/ marshall/index.php). Accessed 23 September 2016.

15. Reese-Cassal K. 2014/2019 Esri Diversity Index, 2014.

16. National Centers for Environmental Information. Metereological versus astronomical seasons. In: National Oceanic and Atmospheric Administration (https://www.ncdc.noaa.gov/news/meteorological-versusastronomical-seasons). Accessed 22 April 2017.
17. Cliff A, Ord K. Testing for spatial autocorrelation among regression residuals. Geographical Analysis 1972; 4(3): 267-284.

18. Ord JK, Getis A. Local spatial autocorrelation statistics: distributional issues and an application. Geographical Analysis 1995; 27(4): 286-306.

19. Epstein D, et al. The effect of neighborhood and individual characteristics on pediatric critical illness. Journal of Community Health 2014; 39(4): 753-759.

20. Feemster KA, et al. Risk of invasive pneumococcal disease varies by neighbourhood characteristics: implications for prevention policies. Epidemiology and Infection 2013; 141(8): 1679-1689.

21. Wang $\mathbf{C}$, et al. Neighborhood income and health outcomes in infants: how do those with complex chronic conditions fare? Archives of Pediatric and Adolescent Medicine 2009; 163(7): 608-615.

22. Yoo JP, Slack KS, Holl JL. Material hardship and the physical health of school-aged children in low-income households. American Journal of Public Health 2009; 99(5): 829-836

23. Beck AF, et al. Inequalities in neighborhood child asthma admission rates and underlying community characteristics in one US county. Journal of Pediatrics 2013; 163(2): 574-580.

24. Gorton CP, Jones JL. Wide geographic variation between Pennsylvania counties in the population rates of hospital admissions for pneumonia among children with and without comorbid chronic conditions. Pediatrics 2006; 117(2): 176-180.

25. Gupta RS, et al. Geographic variability in childhood asthma prevalence in Chicago. Journal of Allergy and Clinical Immunology 2008; 121(3): 639-645.e1.

26. Yousey-Hindes KM, Hadler JL. Neighborhood socioeconomic status and influenza hospitalizations among children: New Haven County, Connecticut, 20032010. American Journal of Public Health 2011; 101(9): 1785-1789.

27. Ghio AJ. Particle exposures and infections. Infection 2014; 42(3): 459-467.

28. Larson K, Halfon N. Family income gradients in the health and health care access of US children. Maternal and Child Health Journal 2010; 14(3): 332-342.

29. Hardt NS, et al. Neighborhood-level hot spot maps to inform delivery of primary care and allocation of social resources. The Permanente Journal 2013; 17(1): 4-9.

30. Ram PK, et al. Household air quality risk factors associated with childhood pneumonia in urban Dhaka, Bangladesh. American Journal of Tropical Medicine and Hygiene 2014; 90(5): 968-975.

31. Kravchenko J, et al. Long-term dynamics of death rates of emphysema, asthma, and pneumonia and improving air quality. International Journal of Chronic Obstructive Pulmonary Disease 2014; 9: 613-627.

32. Ware DN, et al. Household reporting of childhood respiratory health and air pollution in rural Alaska Native communities. International Journal of Circumpolar Health 2014; 73: 1-10.

33. Qiu H, et al. Coarse particulate matter associated with increased risk of emergency hospital admissions for 
pneumonia in Hong Kong. Thorax 2014; 69(11): 10271033.

34. Schwartz J. PM10, ozone, and hospital admissions for the elderly in Minneapolis-St. Paul, Minnesota. Archives of Environmental Health 1994; 49(5): 366-374.

35. Grant WB. The role of geographical ecological studies in identifying diseases linked to UVB exposure and/or vitamin D. Dermatoendocrinology 2016; 8(1): e1137400.

36. Juzeniene A, et al. The seasonality of pandemic and non-pandemic influenzas: the roles of solar radiation and vitamin D. International Journal of Infectious Diseases 2010; 14(12): e1099-e1105.

37. Grant WB, Giovannucci E. The possible roles of solar ultraviolet-B radiation and vitamin $\mathrm{D}$ in reducing case-fatality rates from the 1918-1919 influenza pandemic in the United States. Dermatoendocrinology 2009; 1(4): 215-219.
38. Muhe L, et al. Case-control study of the role of nutritional rickets in the risk of developing pneumonia in Ethiopian children. Lancet 1997; 349(9068): 1801-1804.

39. Wayse V, et al. Association of subclinical vitamin D deficiency with severe acute lower respiratory infection in Indian children under 5 y. European Journal of Clinical Nutrition 2004; 58(4): 563-567.

40. Gunier RB, et al. Traffic density in California: socioeconomic and ethnic differences among potentially exposed children. Journal of Exposure Analysis and Environmental Epidemiology 2003; 13(3): 240-246.

41. Jenkin ME, Clemitshaw KC. Ozone and other secondary photochemical pollutants: chemical processes governing their formation in the planetary boundary layer. Atmospheric Environment 2000; 34(16): 2499 2527. 